\title{
Fluoroquinolones and the risk of serious arrhythmia: a population- based study
}

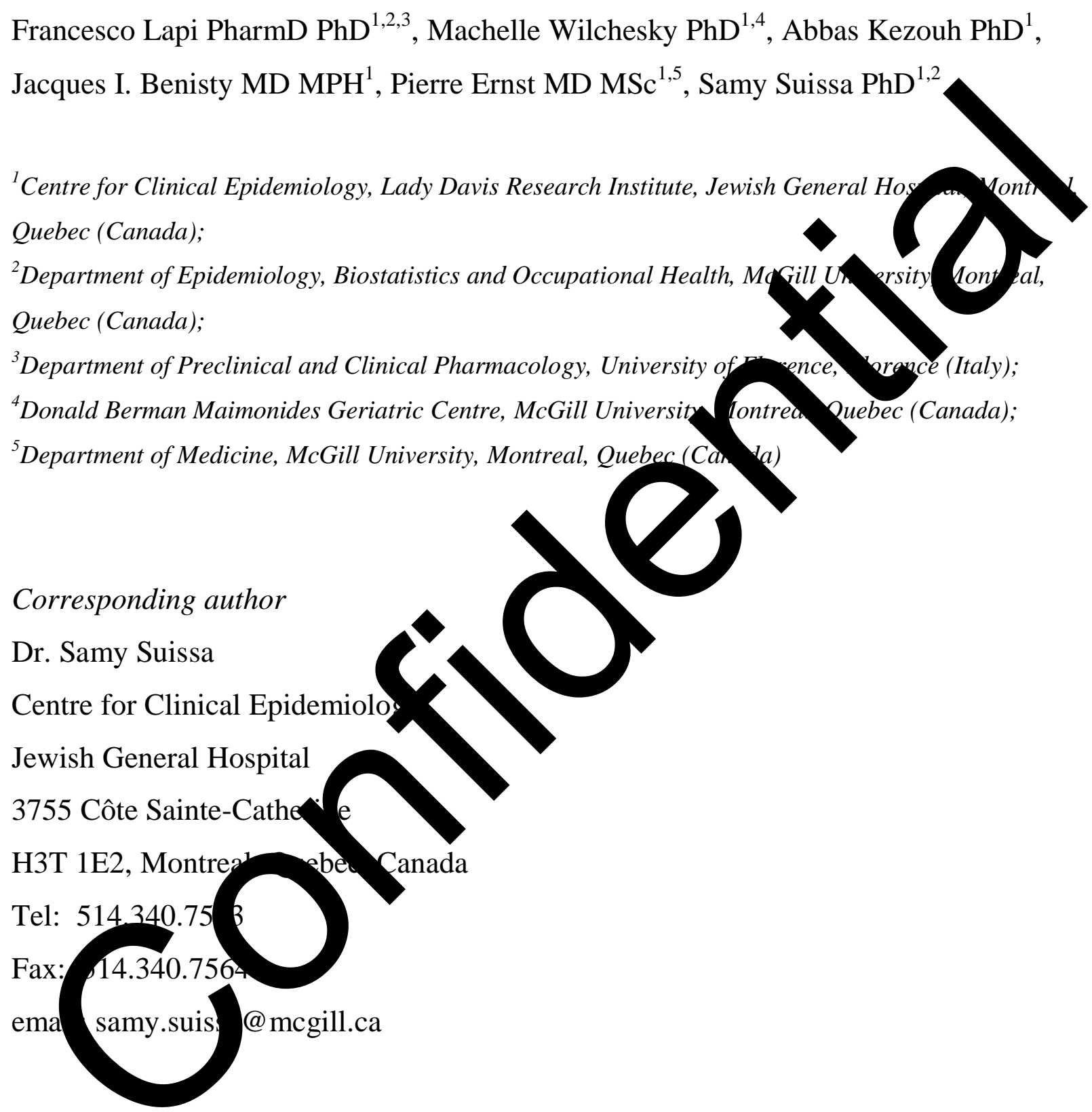

Key words: fluoroquinolones, serious arrhythmia, ventricular arrhythmia, sudden/unattended death, cohort study

Running title: Fluoroquinolones and serious arrhythmia 


\section{Key points:}

Few observational studies have been conducted on fluoroquinolone-related cardiac rhythm disorders. Our results demonstrate that patients newly exposed to fluoroquinolones had a greater risk of developing serious arrhythmias. This effect was due predominantly to gatifloxacin, moxifloxacin and ciprofloxacin.

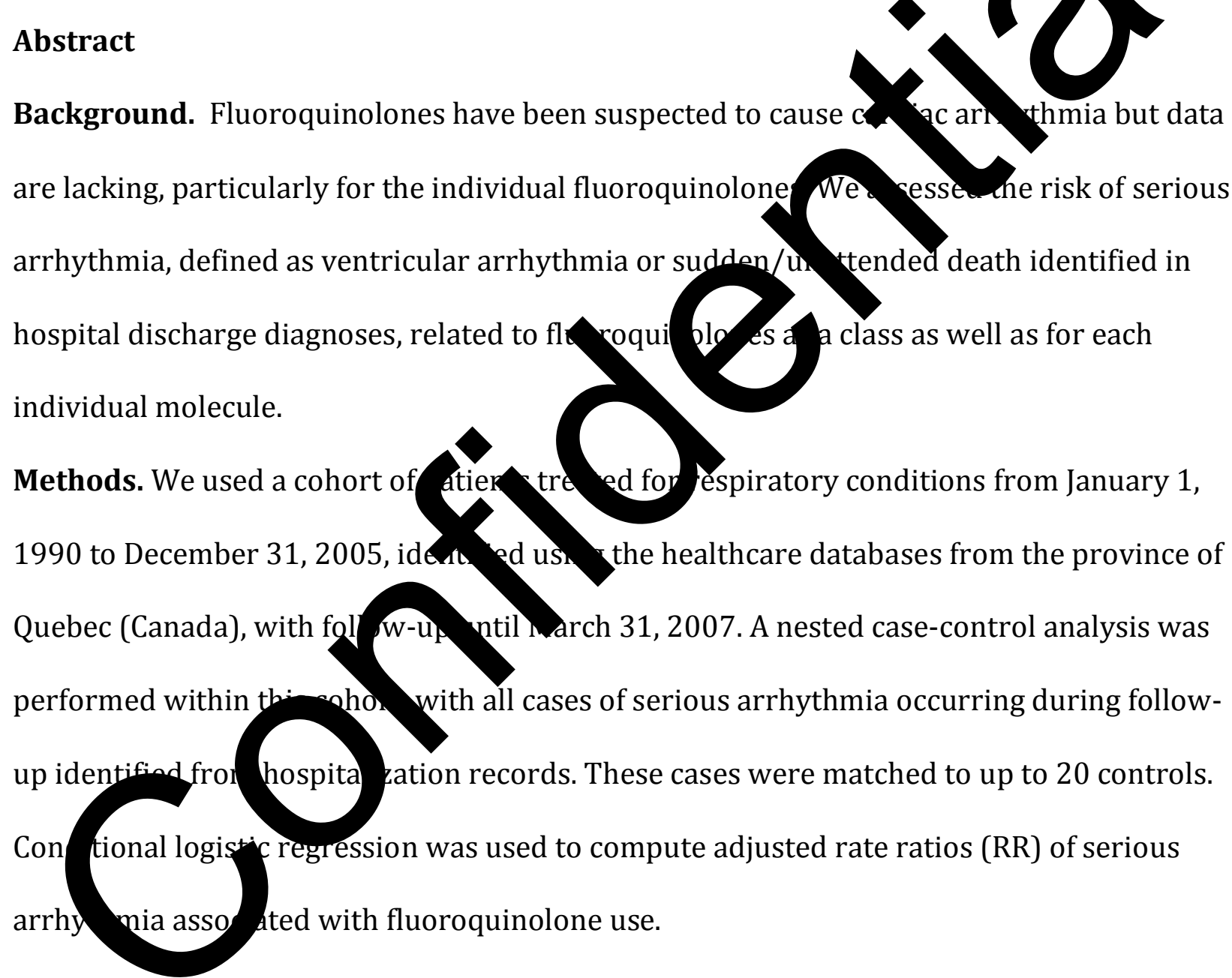

Results. Within the cohort of 605,127 subjects, 1,838 cases were identified (incidence rate $=4.7 / 10,000$ person-years). The rate of serious arrhythmia was elevated with current fluoroquinolone use (RR=1.76; 95\% CI: 1.19-2.59), in particular with new current use $(\mathrm{RR}=2.23 ; 95 \% \mathrm{CI}: 1.31-3.80)$. Gatifloxacin use was associated with the highest rate 
$(\mathrm{RR}=7.38$; 95\% CI: 2.30-23.70); moxifloxacin and ciprofloxacin were also associated with elevated rates of serious arrhythmia $(\mathrm{RR}=3.30$; $95 \% \mathrm{CI}$ : 1.47-7.37 and $\mathrm{RR}=2.15$; $95 \% \mathrm{CI}$ :

\subsection{4-3.46, respectively).}

Conclusions. The use fluoroquinolones is associated with an elevated risk of serious arrhythmia, with some differences among molecules. Given that the individual fluoroquinolones share various indications, the relative risks of
inform the choice of different molecules in high-risk patients.
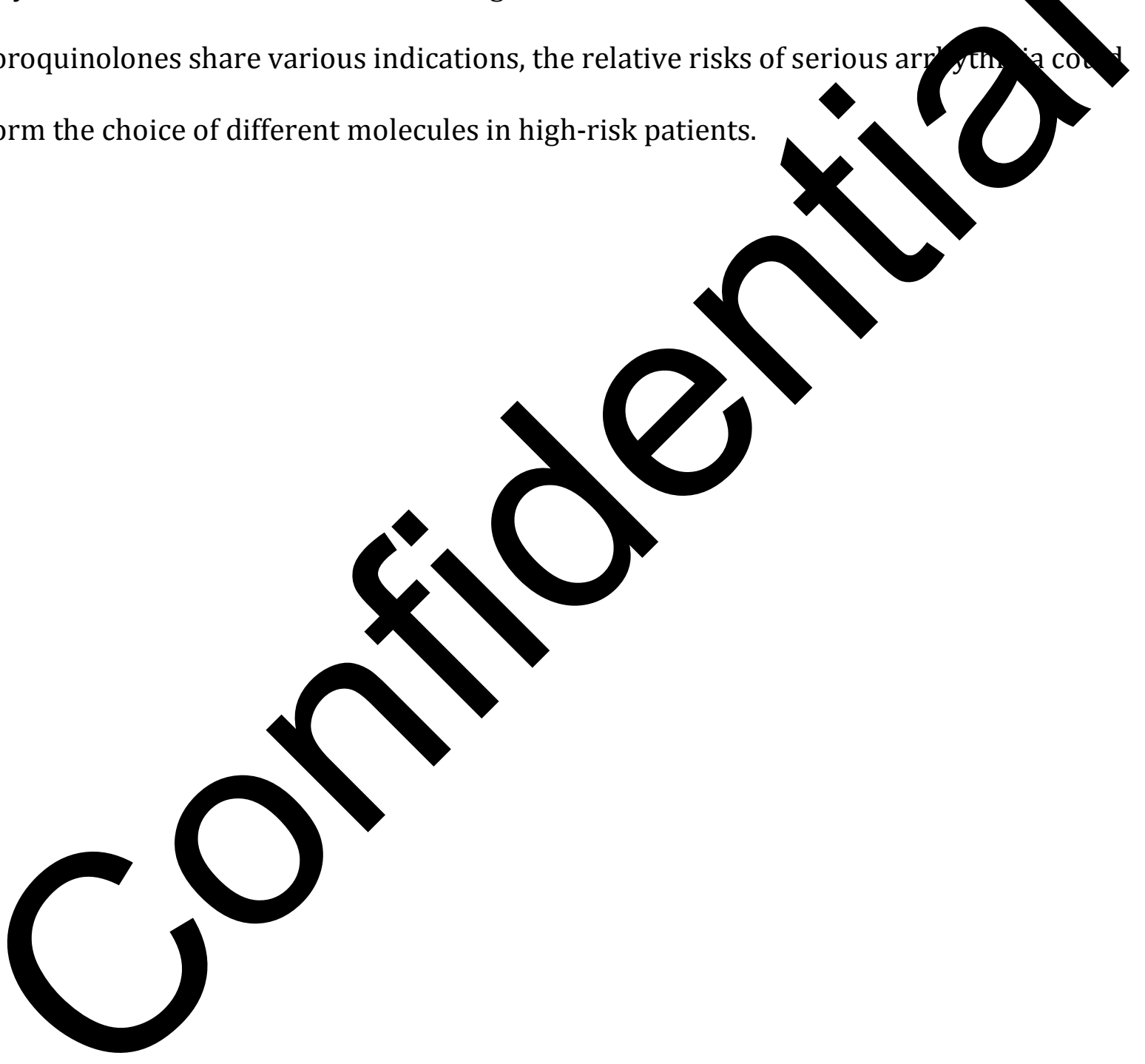


\section{Background}

Fluoroquinolones are antimicrobial drugs frequently used in the treatment of bacterial infections in both primary and secondary care. [1,2] From a clinical viewpoint, fluoroquinolones offer several advantages due to their broad spectrum of activity and a high bioavailability for oral administration. [3, 4] Despite their proven efficacy, hever, the risk/benefit profile of these antibiotics still requires careful evaluation are prescribed. Their association with various adverse drug reactionsuch a QTe nterval prolongation and Torsades de Pointes which can lead to ventricum fibrills on, cardiac arrest and sudden cardiac death,[5] has resulted in revisin the icatiunfor use of some fluoroquinolones (i.e., moxifloxacin) while certain antibiotio $n$ this class have been withdrawn from the European and US mats en
Establishing a causal lir
been difficult owing to the rarit, To date, only one protion sased study has been conducted, reporting a more than 3 -fold higher riol- de de loping ntricular arrhythmia and cardiac arrest with the use of fluo quinolone 11 This study, however, did not evaluate the risk separately for the as the occurrence of exacerbations of chronic respiratory disease. [12, 13]

In this study, we assessed the risk of serious arrhythmia related to fluoroquinolones as a class as well as for each individual fluroquinolone antibiotic. 


\section{Methods}

\section{Data source}

Data were drawn from the RAMQ and MedEcho databases, for which demographic and healthcare utilization information are currently collected for all 7 million of residents of the province of Quebec (Canada).

Before January 1, 1997, the RAMQ prescription drug insurance plan residents who were either 65 years and older, or welfare recipients and their fild en. After this date, the drug plan was modified to give access to empor rests and their spouse/children who did not have access to alternate priva dro insulunce coverage.[14] The RAMQ database also provides the dates of death The M Echo database contains information pertaining to all hospital adm vions the pital deaths in Quebec obtained from the data recorded by archivists on th nos ralizanun discharge summary sheet. These databases have been prous use con uct epidemiological studies. [14-16] The Research Ethics Conm an of Jewish General Hospital has granted approval for this protocol. The prot col no ber 11-039.

\section{Cohort dafinjtio}

We ed a cohort paulents $(n=1,410,211)$ treated for respiratory conditions from January 1, 19 to Decen er 31, 2005, identified using the RAMQ database, with follow-up until March 31, 2007. Respiratory medications comprised bronchodilators, nasal or orallyinhaled corticosteroids, and anti-asthma medications (cromoglycate, nedocromil, montelukast, zafirkulast, and ketotifen). 
Subjects with fewer than two complete years of RAMQ coverage before the date of their first respiratory medication prescription were excluded from the study. Thus, the date of cohort entry (t0) occurred 730 days after a patient's first eligible day of coverage, assuming that they were not excluded at any point in time between day 0 and day 729 . Subjects were excluded if they had a previous history of arrhythmia before or on day of cohort entry based on the identification of codes for hospitalization (i.e., ve atrial arrhythmia at any diagnosis position, primary or otherwise) or edica ervi es (i.e., arrhythmia-related surgical/interventional procedures) or on rated us of plan, had an event of serious arrhythmia, death (if notregis as as sudden or unattended death in MedEcho) or until March 31, 200 the e tollow-up) whichever came first.

\section{Case definition}

Cases of serious arrhythm , den as antricular arrhythmia or sudden/unattended death, were identifi-ang D-9 and ICD-10 codes (primary diagnosis position) for hospitalimetigns. (rst, dia oses of paroxysmal ventricular tachycardia, ventricular fibri ation and/o flutter, cardiac arrest $[11,17,18]$ other unspecified forms of arrhythmia diagnosis codes were then coupled for each individual with specific cardiac surgical/interventional procedures (i.e. implantation, replacement or removal of cardioverter-defibrillator; ventricular tachycardia ablation; electrophysiological studies) as well as pharmacological treatments (i.e. beta-blockers, mexiletine and magnesium). [19] 
Codes pertaining to cardiac interventions used in the treatment of rhythm disorders $( \pm 3$ months from the date of diagnosis) identified within the hospitalization data and all prescriptions for an antiarrhythmic drug ( \pm 1 months from the date of diagnosis), were examined with respect to each acute event of serious arrhythmia. The index date was then defined as the earlier date from either hospital admission (or the date of death) the occurrence of a surgical/interventional procedure or pharmacological trea

\section{Exposure definition}

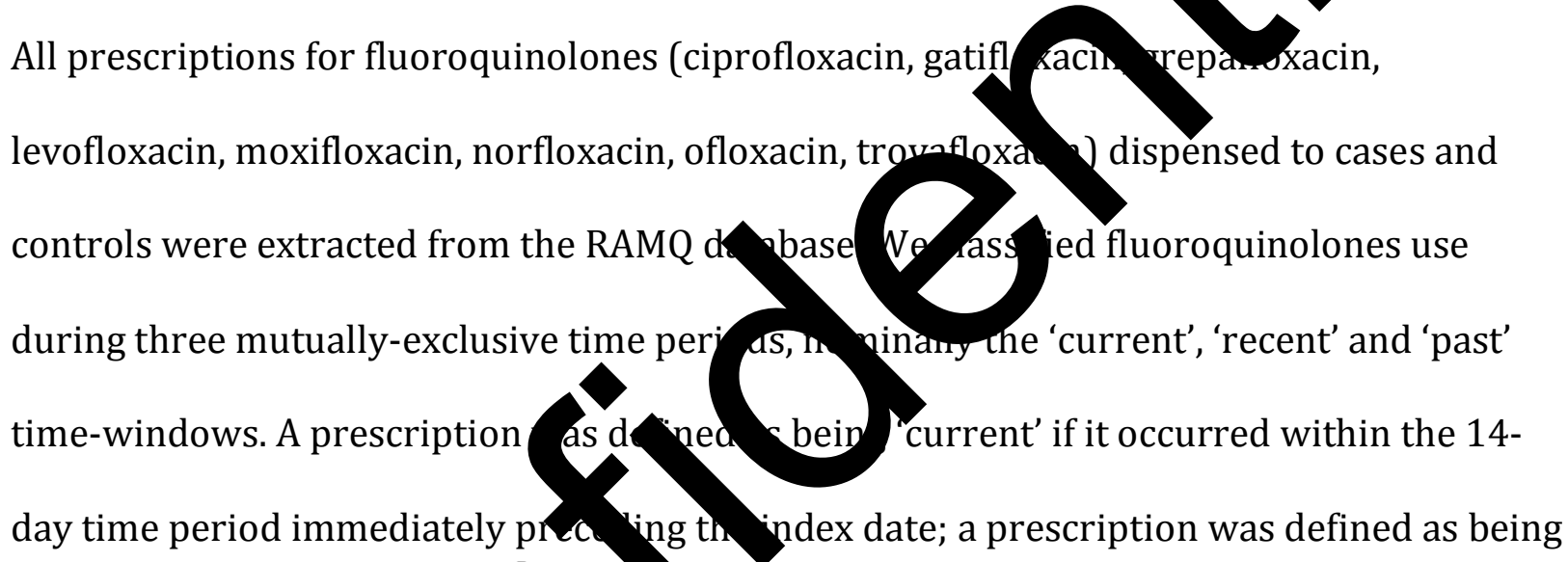
'recent' if it occurred at an poin luring the 15-30 day time-window before the index date; those prescriptions arde consideraros 'po 'prescl tions. Finally, current use was further classified as being use whi (was either new or "not new" depending on whether or not they had also been recen sers. 'Cu ent' (either 'new' or 'not new'), 'recent' and 'past' use was then compared with 'no use' of fluoroquinolones.

\section{Covariates}


Variables pertaining to risk factors for serious arrhythmia as well as those identified as being potential confounders of the fluoroquinolone-arrhythmia association were identified from the RAMQ and MedEcho databases. Specifically, comorbidities such as congestive heart failure, valvular disease, coronary artery disease, enlarged heart, congenital structural cardiovascular abnormalities, other cardiovascular diseases, atherosch osis, hyperlipidemia, chronic and/or acute renal failure, diabetes mellitus (diag prescriptions of antidiabetic drugs), respiratory diseases, anemia, thy id dis ases septicemia and the overall number of hospitalizations were capt in thear before the index date. Additionally, concurrent use of various medicat on ches sturn as antihypertensives (beta blockers, calcium channel blockers, CE Inhibitors, angiotensin

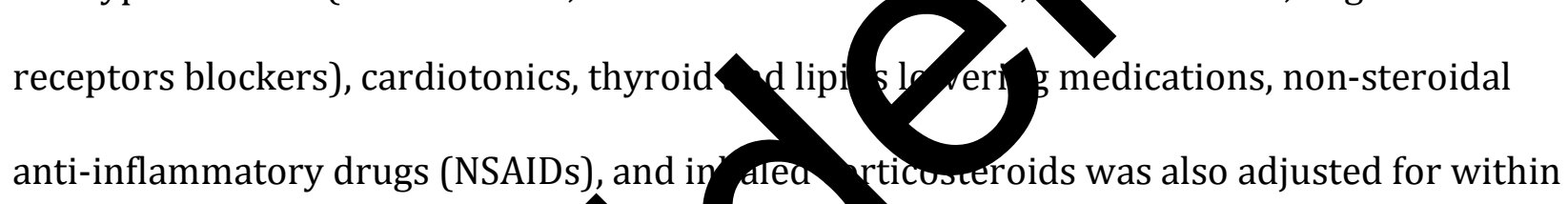
the same time-window.

Given the fact that COPD $\mathrm{x}$ erbac $\mathrm{s}$ are both an independent risk factor for serious arrhythmia $[12,1$ and well otentially associated with our exposure, we considered exacerb on thring during the 30 day time-window leading to the index date as namible sfound 'Moderate' exacerbations were identified when a 'serious' exacerbation was defined as a primary diagnosis of hospitalization for COPD.[20] Finally, prescriptions for medications filled within 30 days before the index date which can potentially induce arrhythmia (i.e. antiarrhythmic drugs (if not used to define cases), macrolides, pirimetamine, sulfamethoxazole/trimethoprim (if not used to define 
'moderate' exacerbation), antiviral and antifungal medications, antidepressants, other central nervous systems (CNS) drugs, antiemetic drugs, and antimigraine medications) [21, 22] were included and adjusted for in our multivariate models.

\section{Nested case-control analysis}

Up to 20 controls per case were randomly selected within each risk set. Eli who were alive and event-free on the calendar date of the serious arreythmi case the index date) were matched to their respective cases on age ( \pm 5 y sex, cohort year of entry. Matched controls were assigned the same index d eas ropective case. 'Current use', 'recent use', 'past use' and 'no use' of flınroqu, lones was compared between cases and their matched controls, nd th as cian between serious arrhythmia and fluoroquinolone use was estimated.

\section{Data analysis}

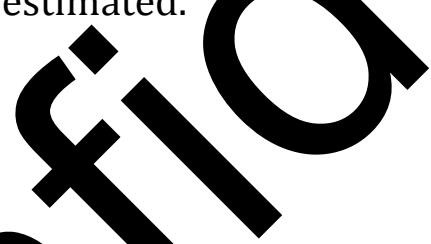

The incidence density for re ou me interest was computed in the cohort. Conditional logistic regression compute odds ratios of serious arrhythmia which, for the and Catio (RR) and the related 95\% confidence intervals (CI). [23] In addition to age, sex adjusted for the risk factors and confounders described above. Furthermore, we computed the attributable risk of serious arrhythmia due to fluoroquinolones by applying the RRs to the background incidence rate derived from our cohort. 
Three sensitivity analyses were conducted in order to further clarify our results.

Firstly, we controlled for immeasurable time bias [24] by repeating the analyses after excluding patients who had been hospitalized during the current time-window. Secondly, the definition of 'current' use was modified by varying the duration of the 'current' timewindow from 14 to 21 and 28 days. Finally, all models were re-run using a more definition whereby cases identified via diagnoses pertaining to "unspecific rin wia or "sudden/unattended death" were restricted to only those which wereveced a or ollowed by cardiac surgical/interventional procedures or pharmacologic vatmo Statistical analyses were conducted using SAS version 9.2 TS2M3 (SA Anst te Int, Cary, North Carolina).

\section{Results} serious arrhythmia were identike iving incidence rate equal to 4.7 per 10,000 personyears at risk (Figure 1).

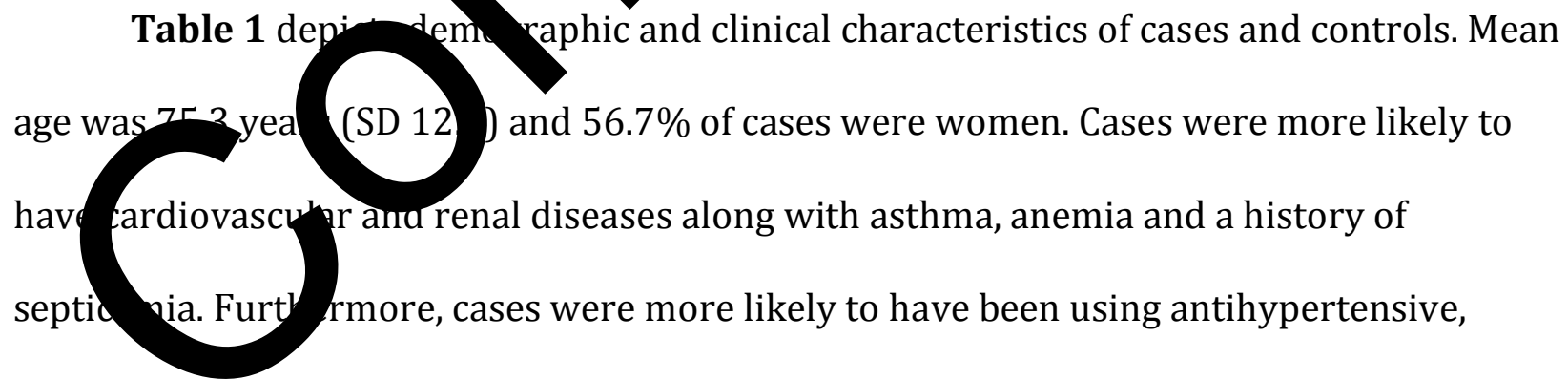
cardiotonics, or lipid lowering drugs as well as NSAIDs and respiratory medications.

Frequency of hospitalizations for COPD exacerbations were also proportionally higher among cases than controls ( $7.3 \%$ vs. $1.5 \%)$, as well as the use of medications known to be potentially pro-arrhythmic. As shown in Table 2, most of the cases were non-fatal. Overall, 
121 and 272 cases were combined with a surgical/interventional procedure or pharmacotherapy, respectively.

Table 3 shows that, when the exposure to fluoroquinolones was modeled according to recency of use, the adjusted RR was not significantly elevated among current ( $R R=1.34$; 95\% CI: 0.92-1.93), recent (RR=1.24; 95\% CI: 0.82-1.86), or past $(\mathrm{RR}=1.14 ; 95 \%$ 1.32) users. When current use was further broken down into current use and "not new", patients newly exposed appeared at greater risk (RR= $62 ; 950 \mathrm{CI}$. 0.97 2.71) than those who had received these medications in both cul and time periods; although again, these RRs were not statistically sif ifica dy diserent from no increase in risk. Among individual fluoroquinolones, antiflo. in was the only molecule to be significantly associated with a higher of se ov arr thmia (RR=3.97; 95\% CI: 1.1513.62).

To account for immeas able ne s, we mited the analysis to those patients who were not hospitalized during cun time-window. In this analysis, the overall risk of serious arrhythmia was $\mathrm{S}$ vifica cly higher among current users (RR=1.76; 95\% CI: 1.19-2.59) as comp wit ecent and past users. Furthermore, "new" current users use hich was " $p$ t new. After stratifying our analyses by individual fluoroquinolone mole vas, gatifl acin reported the highest point estimate (RR=7.38; 95\% CI: 2.30-23.70) while moxifloxacin and ciprofloxacin were also associated with a significantly increased risk of serious arrhythmia (RR=3.30; 95\% CI: $1.47-7.37$ and $R R=2.15$; 95\% CI: 1.34-3.46, respectively; Table 4). The additional cases of serious arrhythmia for the "new" current 
users were 5.8 per 10,000 person-years, while moxifloxacin, ciprofloxacin and gatifloxacin reported an attributable risk of 10.8, 5.4 and 30 per 10,000 person-years, respectively.

When we varied the duration of the current time-window from 14 to 21 and 28 days, the overall risk of serious arrhythmia among users of fluoroquinolones, decreased (i.e., 21 days: adjusted RR=2.10; 95\% CI: 1.53-2.88; for 28 days: adjusted RR=1.9. 95\% CI:

Finally, sensitivity analyses using the more strict case definitio still p ovid d consistent results (adjusted RR for current users was 1.65, 95\% 03-2.

\section{Discussion}
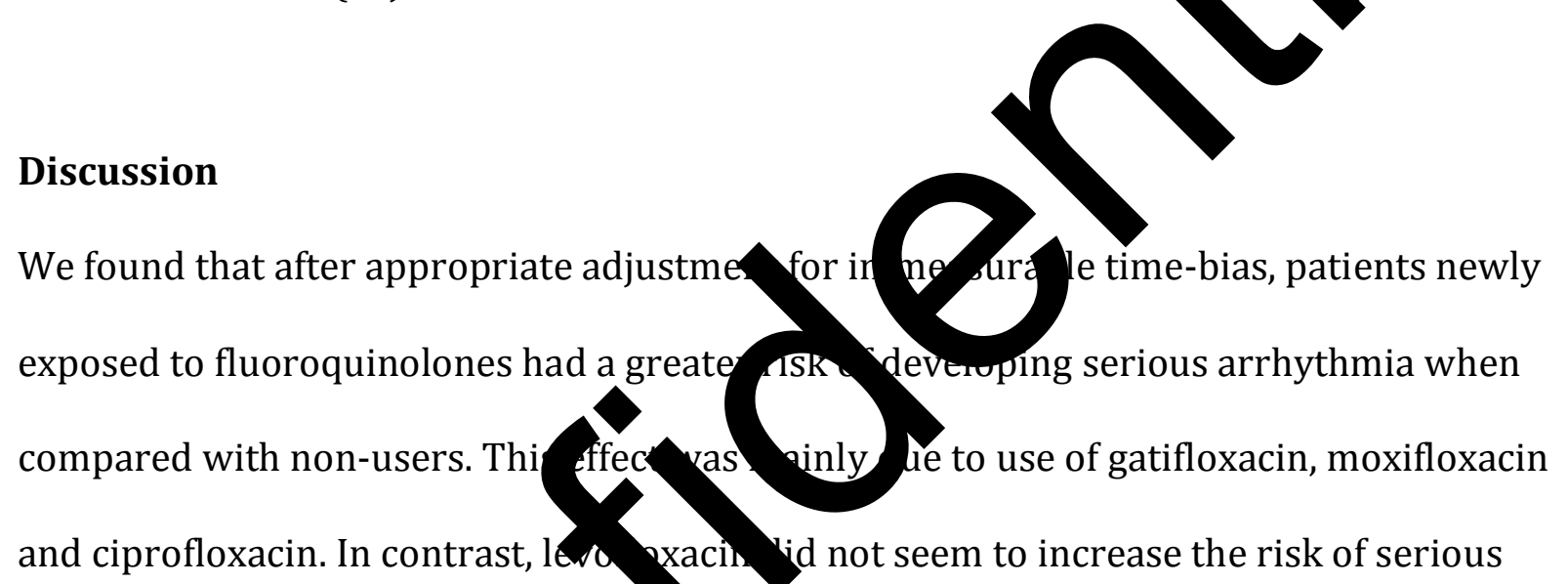
arrhythmia.

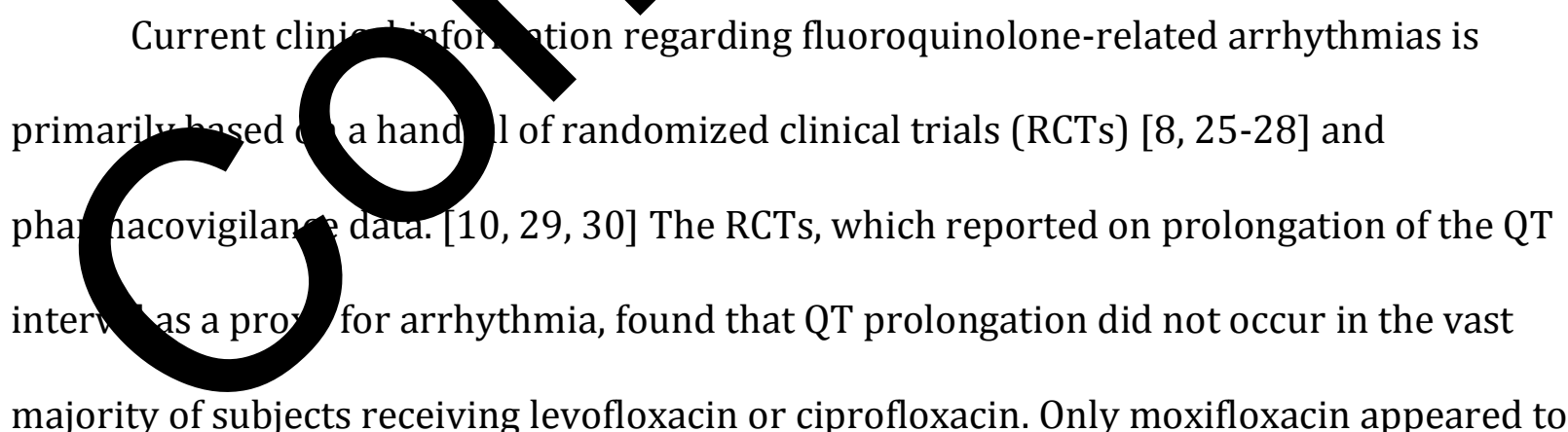
be associated with a potentially clinically-significant prolongation of the QT interval. [8, 2527] On the other hand, the most recent pharmacovigilance analyses [10, 29] raised 
concerns of an excess of Torsades de Pointes among moxifloxacin, [10, 29] gatifloxacin, [10] levofloxacin, [10,29] and ciprofloxacin [29] users.

RCTs provide only limited information on the "real world use" of such medications, because they most often exclude subjects with significant co-morbidities. Pharmacovigilance data, on the other hand, is limited by selective reporting. Hem observational studies are needed to complement these findings. [31]

To date, only one other observational study on the ventricular rrhyth fia g cardiac arrest risk associated with fluoroquinolones has been conducted houg n elevated risk was found, we believe that this study by Zambon and cowg Rers 1] haseveral limitations which we have tried to address. First, we adopte shorter time-window for cardiac rhythm. We were also able to exarent exposure, to be more consistent when confounder of the association ond quino wes with ventricular arrhythmia. [12,13] Our results further emo rate ne importance of immeasurable time bias [24] in this specific context aris bres when a study includes exposure times during periods where exancyre nnot be heasured. Given that the RAMQ database (similarly to many heal administr ive uatabases), does not include information on medications dispensed to ho - tal inpat hts, patients would incorrectly appear as unexposed during hospitalizations which could incidentally occur during the etiological time period under study.

With respect to the analyses performed to identify risks associated with the individual fluoroquinolone molecules, a greater effect size was expectedly higher for 
moxifloxacin. Interestingly, it was gatifloxacin, a drug that was withdrawn in 2006 due to serious hyperglycemic events, [32] which we found to be associated with the highest risk of arrhythmia. These results on moxifloxacin and gatifloxacin are in keeping with previous pre-clinical findings [33] and pharmacovigilance reports. [10, 29] Furthermore, our findings confirm the EMA report [34] which assessed a 'low potential' for arrhyt ia with levofloxacin. Finally, this study adds new information on the serious arrhy associated with the use of ciprofloxacin, whose previous data were le consi ent fan for other fluoroquinolones. [29, 30, 35]

There is a sound biologic rationale for the associatic bet frowoninolone use and serious arrhythmias. Cardiac QT interval is regulated by interplay of ionic currents through various ion channels, and its prol gatio caleg herate into Torsades de Pointes, which might be followed by ventricular fi Mllat 7 , carturac arrest and sudden death. Fluoroquinolones are able to $\mathrm{p}$ lons anter al by interfering with the electrophysiological function of sol siun bannels that contribute to the regulation of action potentials in the cas riac These changes can result in ventricular arrhythmias. Therefore, the risk revendio scular events with fluoroquinolones depends on the degree to whichnan $\sin$ molec inhibits the potassium channels. [5, 33] Chemically, although the (bstitution postuon 5 (as per moxifloxacin and gatifloxacin) of the quinolone nucle seems to ffect cardiotoxicity, researchers have not yet identified a definite structural moiety that significantly increases the QT prolongation, so differentiating the risk profile.[3] Along these lines, our results indicated a greater risk of serious arrhythmia for ciprofloxacin which does not carry any functional group in position 5 . It is possible that genetic variants [36] acting as modifiers in the susceptibility to rhythm disorders, may be 
associated with the risk of individual molecules, $[37,38]$ and this would require further research.

This study has several limitations. Firstly, the diagnoses of serious arrhythmia have not been formally validated. However, misclassification of the outcome should not differ according to use of fluoroquinolones, and therefore such misclassification can be pected to reduce the association suggesting that risks may be underestimated in $t$ In addition, after operationally modifying the event definition as a sepitivity naly is so that a more strict definition of cases was used for the diagnoses anecit rrhythmias" and "sudden/unattended death", we observed similar resu s wh regareto the RR of serious arrhythmia, which suggests that misclassification wa not a significant issue. Furthermore the overall incidence rate of out whe in line with published estimates. [39] Secondly, it is possible tha cesit con counding may still play a role and partially bias our results. How er, the estimates of effect, it would be hard to imagine how greater ability ye to residual confounders could explain the elevated risks associated th the omoxifloxacin, gatifloxacin and ciprofloxacin. Thirdly, the small nuer o yposed subjects prevented the definition of an etiologicallyrelevant angur time-wy dow shorter than 2 weeks which might also have resulted in an und estimation the true effect. However, the progressive risk reduction that we obser after ending the exposure time-window confirms a biologically plausible acute effect. Fourthly, we were not able to estimate the difference in risk of serious arrhythmia between new and ongoing use for the individual fluoroquinolone molecules due to a lack of statistical power. Finally, given our study cohort, the generalizability of our findings may be limited to populations treated at least once with a respiratory medication. 
From a public health perspective, the use of these antibiotics, especially ciprofloxacin and levofloxacin, has been increasing in the last decades. [2, 40] As a consequence, it is possible that we may observe a corresponding increase in the number of serious and unintended adverse effects. Given that the individual fluoroquinolones share various indications, the relative risks of serious arrhythmia could inform the cho of molecules in high-risk patients. Given that serious arrhythmias are rare, fy conducted in a larger population of patients with these outcomes wo be befic Min order to provide confirmation of our findings.

\section{Acknowledgements}

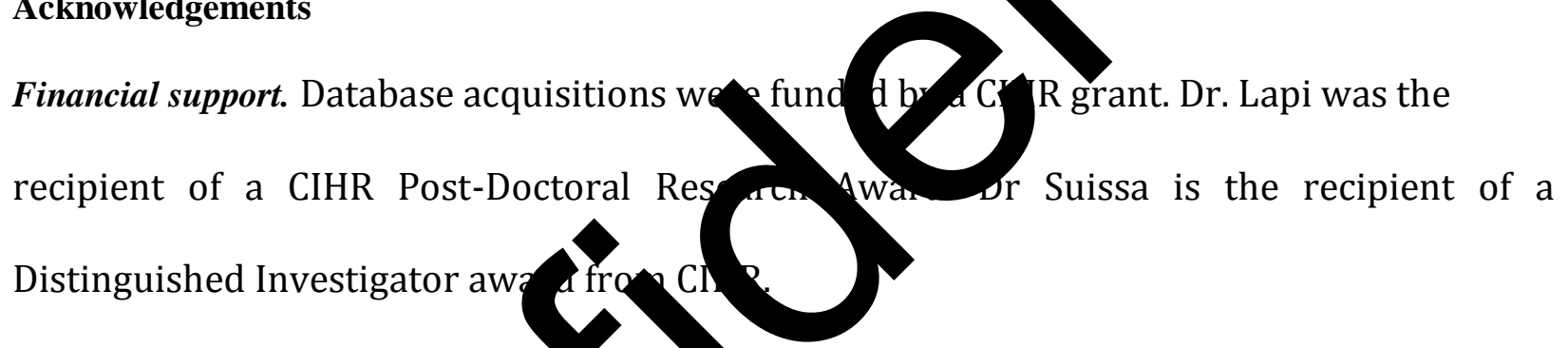

Potential Conflict of Interests. Dr. \& Boehringer Ingelheim and axo nith ne, and has participated in advisory board meetings and as spealaar in nferences for AstraZeneca, Boehringer-Ingelheim, GlaxoSmithKline Jovartis fizer and Merck. Dr. Ernst has received speaker fees and has
atte ed advisory borarers for AstraZeneca, Boehringer Ingelheim, GlaxoSmithKline, Merck,
Nova and Ny med. Dr. Lapi, Dr. Wilchesky, Dr. Kezouh and Dr. Benisty do not have any conflicts of interest to disclose. 


\section{References}

1. Owens RC, Jr., Ambrose PG. Clinical use of the fluoroquinolones. Med Clin Non Am 2000; 84:1447-1469.

2. Ferech M, Coenen S, Malhotra-Kumar S, et al. European St crobial Consumption (ESAC): outpatient quinolone use in Europe Ant icrob hemother 2006; 58:423-427.

3. Mehlhorn AJ, Brown DA. Safety concerns wit mo quit gnes. Ann Pharmacother 2007; 41:1859-1866.

4. Owens RC, Jr., Ambrose PG Antim robial fety: focus on fluoroquinolones. Clin Infect Dis 2005; 41 Supp 5.544 .57$.

5. Owens RC, Jr., Ambrose PG. rsadesde pointes associated with fluoroquinolones. Pharmacotherapy 2; 22:0 668; discussion 668-672.

6. Owens RC

7.

Diagn Mio biol Infect Dis 2005; 51:45-49.

7.

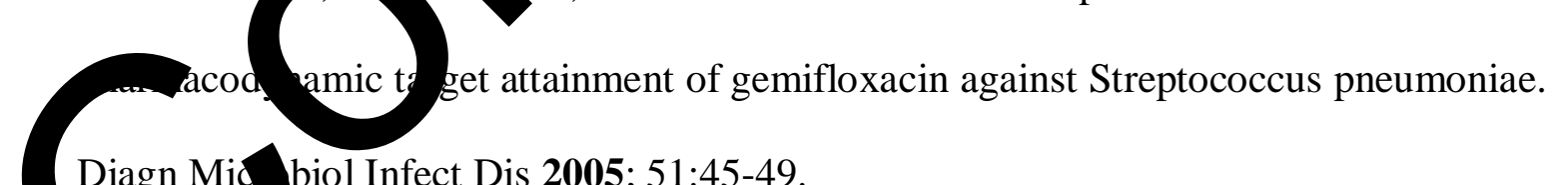

HH profile of the fluoroquinolones: focus on levofloxacin. Drug Saf 2010;

\section{3:353-369.}

8. Pugi A, Longo L, Bartoloni A, et al. Cardiovascular and metabolic safety profiles of the fluoroquinolones. Expert Opinion on Drug Safety 2011. 
9. Lapi F, Tuccori M, Motola D, et al. Safety profile of the fluoroquinolones: analysis of adverse drug reactions in relation to prescription data using four regional pharmacovigilance databases in Italy. Drug Saf 2010; 33:789-799.

10. Frothingham R. Rates of torsades de pointes associated with ciprofloxacin, ofloxacin, levofloxacin, gatifloxacin, and moxifloxacin. Pharmacotherapy 2001; 21:1460 472.

11. Zambon A, Polo Friz H, Contiero P, Corrao G. Effect of macrolide and antibacterials on the risk of ventricular arrhythmia and cardiac arrect: an of erva onal study in Italy using case-control, case-crossover and case-tim ontro signs. Drug Saf 2009; 32:159-167.

12. Schumaker GL, Epstein SK. Managing acute respirato, Gilure during exacerbation of chronic obstructive pulmonary diseasa Resp Car 10 19y; 496-782.

13. Khokhar N. Cardiac arrhythmias asso respiratory failure in chronic obstructive pulmonary dis se. Milit Med 1 ; $;$ 146:856-858.

14. Tamblyn R, Lavoie G, Pet $\mathrm{L}, \mathrm{M}$ vette J. The use of prescription claims databases in pharmacoepidemiol the accuracy and comprehensiveness of the prescription claims \& base in Quebec. J Clin Epidemiol 1995; 48:999-1009.

15. Suissa $S$, zouh A zrns $\mathrm{P}$. Inhaled corticosteroids and the risks of diabetes onset and progression. $1 \mathrm{~J}$ Med 2010; 123:1001-1006.

16. Freeman E Roy-Gagnon MH, Fortin E, Gauthier D, Popescu M, Boisjoly H. Rate of enton nalmitis after cataract surgery in quebec, Canada, 1996-2005. Arch Ophthalmol 2010; $128: 230-234$.

17. Staffa JA, Jones JK, Gable CB, Verspeelt JP, Amery WK. Risk of selected serious cardiac events among new users of antihistamines. Clin Ther 1995; 17:1062-1077. 
18. Hennessy S, Leonard CE, Freeman CP, et al. Validation of diagnostic codes for outpatient-originating sudden cardiac death and ventricular arrhythmia in Medicaid and Medicare claims data. Pharmacoepidemiol Drug Saf 2010; 19:555-562.

19. Zipes DP, Camm AJ, Borggrefe M, et al. ACC/AHA/ESC 2006 guidelines for management of patients with ventricular arrhythmias and the prevention of su cardiac death: a report of the American College of Cardiology/America Association Task Force and the European Society of Cardiology Comnutt for ructice Guidelines (Writing Committee to Develop guidelines for mement parients with ventricular arrhythmias and the prevention of sudden ce atave eloped in collaboration with the European Heart Rhythm Assocka n and the Heart Rhythm Society. Europace European paine Society. Europace : European pacing arrhyth fias ndardiac electrophysiology : journal of the working groups on card: cardiac cellular electrophysiology of the Fopean S vety of diology 2006; 8:746-837.

20. de Melo MN, Ernst P, Suis S. Inh d corticosteroids and the risk of a first exacerbation in COPD patients. E ir J 2 4; 23:692-697.

21. SADS. http://www. J.org.ukabout_sads.htm. [last access March 2012].

22. Ray WA, urray K Har K, Arbogast PG, Stein CM. Azithromycin and the risk of cardiovasculan. New Engl J Med 2012; 366:1881-1890.

23. Breslow N Statistics in epidemiology: the case-control study. Journal of the American
Stan Association 1996; 91:14-28.

24. Suissa S. Immeasurable time bias in observational studies of drug effects on mortality. Am J Epidemiol 2008; 168:329-335. 
25. Demolis JL, Kubitza D, Tenneze L, Funck-Brentano C. Effect of a single oral dose of moxifloxacin (400 mg and $800 \mathrm{mg}$ ) on ventricular repolarization in healthy subjects. Clin Pharmacol Ther 2000; 68:658-666.

26. Noel GJ, Goodman DB, Chien S, Solanki B, Padmanabhan M, Natarajan J. Measuring the effects of supratherapeutic doses of levofloxacin on healthy volunteers us four methods of QT correction and periodic and continuous ECG recordings Pharmacol 2004; 44:464-473.

27. Noel GJ, Natarajan J, Chien S, Hunt TL, Goodman DB, Abe Effec of tnree fluoroquinolones on QT interval in healthy adults after 190 2003; 73:292-303.

28. Morganroth J, Dimarco JP, Anzueto Nied mar Choudhri S. A randomized trial comparing the cardiac rhythm safety hospitalized with commun y-acquir pneum ma. Chest 2005; 128:3398-3406.

29. Poluzzi E, Raschi E, Moto Mo ti U, De Ponti F. Antimicrobials and the risk of torsades de pointes: rrom data mining of the US FDA Adverse Event Reporting System. D g Saf 2uro; 33:303-314.

30. Clark DW Cayton Wiron LV, Pearce GL, Shakir SA. Profiles of hepatic and dysrhythmic ascular events following use of fluoroquinolone antibacterials: experience om large cohorts from the Drug Safety Research Unit Prescription-Event Mun ing database. Drug Saf 2001; 24:1143-1154.

31. Suissa S, Garbe E. Primer: administrative health databases in observational studies of drug effects--advantages and disadvantages. Nat Clin Pract Rheumatol 2007; 3:725-732. 
32. Juurlink DN, Park-Wyllie LY, Kapral MK. The effect of publication on Internet-based solicitation of personal-injury litigants. CMAJ 2007; 177:1369-1370.

33. Kang J, Wang L, Chen XL, Triggle DJ, Rampe D. Interactions of a series of fluoroquinolone antibacterial drugs with the human cardiac K+ channel HERG. Mol Pharmacol 2001; 59:122-126.

34. EMEA/CHMP/PhVWP/810358. Pharmacovigilance Working Party (P http://www.ema.europa.eu/docs/en_GB/document_library/Report/201 Tro1 CC5 pr0045 9.pdf [last access May 2012].

35. Knorr JP, Moshfeghi M, Sokoloski MC. Ciprofloxacin cut Q- crval prolongation. Am J Health Syst Pharm 2008; 65:547-5

36. Kaab S, Crawford DC, Sinner MF, etr. A L ge Cnd ate Gene Survey Identifies the KCNE1 D85N Polymorphism as a Po of Drug-Induced Torsades de Pointes. Circ Cardiovasc net 2011 5:91-99

37. Yang Y, Liang B, Liu J, et Iden ration of a Kir3.4 Mutation in Congenital Long QT Syndrome. Am Joury 2010.

38. Paulussen AD, Gilis RA, Amstrong M, et al. Genetic variations of KCNQ1, KCNH2, SCN5A, K NE1, a KCNE2 in drug-induced long QT syndrome patients.J Mol Med

39. 2004; $82: 18$

9. Cobb LA, hos, ventricular fibrillation, 1980-2000. JAMA 2002; 288:3008-3013.

40. Glass SK, Pearl DL, McEwen SA, Finley R. A province-level risk factor analysis of fluoroquinolone consumption patterns in Canada (2000-06). J Antimicrob Chemother 2010; 65:2019-2027. 
Table 1. Comparison of cases of serious arrhythmia and their matched controls.

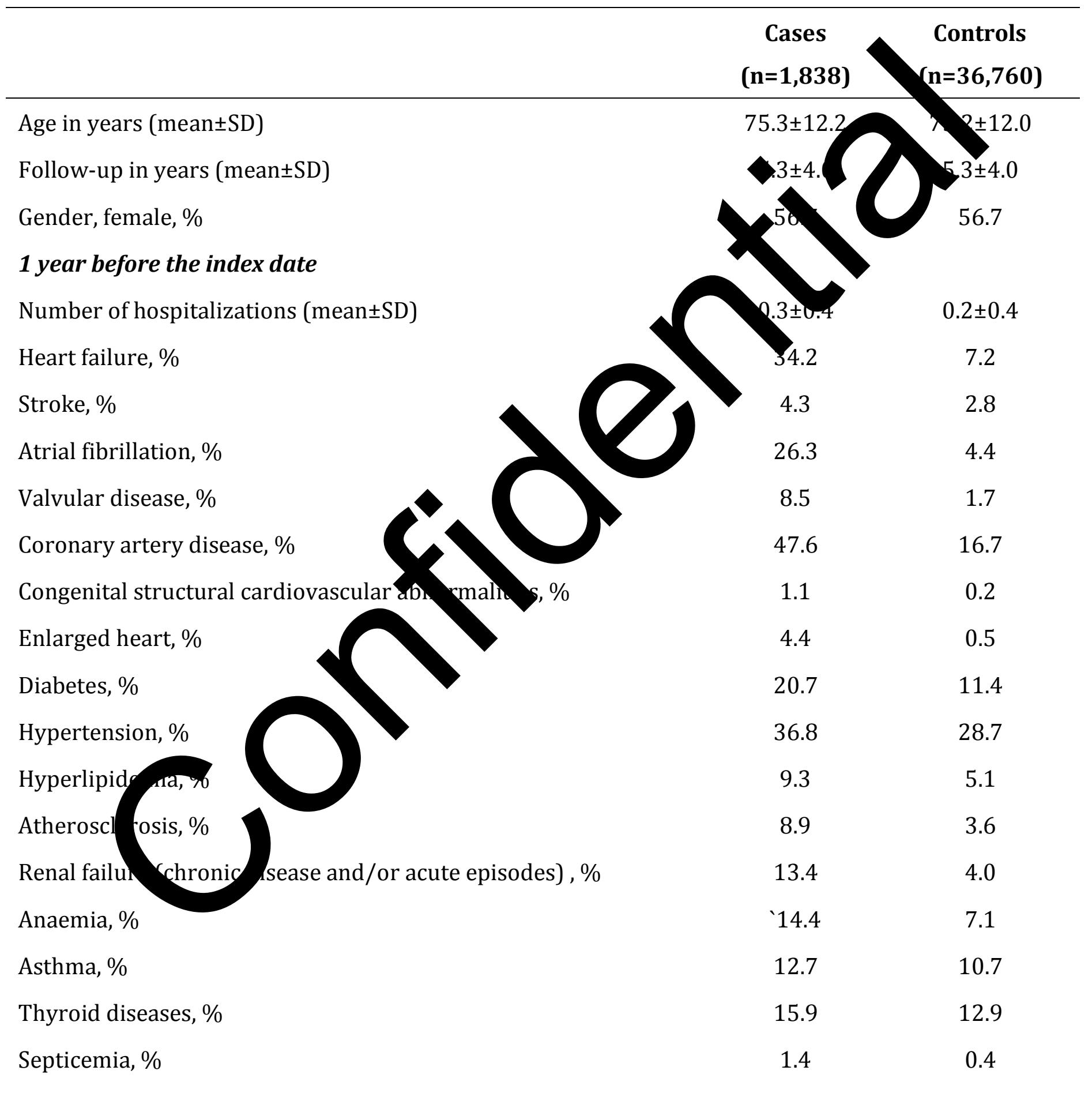

Comedications 


\begin{tabular}{|c|c|c|}
\hline & $\begin{array}{c}\text { Cases } \\
(n=1838)\end{array}$ & $\begin{array}{l}\text { Controls } \\
(n=36,760)\end{array}$ \\
\hline Cardiotonic drugs, $\%$ & 30.6 & 8.3 \\
\hline Oral corticosteroids, $\%$ & 17.7 & 12.2 \\
\hline Inhaled corticosteroids, $\%$ & 36.5 & \\
\hline Other respiratory medications, $\%$ & & \\
\hline Lipid lowering drugs, $\%$ & & \\
\hline Thyroid medications, $\%$ & & 12.1 \\
\hline NSAIDs, \% & & 47.3 \\
\hline 30 days before the index date & & \\
\hline COPD exacerbation, $\%$ & & \\
\hline Severe (COPD hospitalizations) & 7.3 & 1.5 \\
\hline Moderate* & & \\
\hline Macrolide and OC & 0.05 & 0.08 \\
\hline Non-mac & 1.0 & 0.6 \\
\hline Antiarrhythmic d & 5.5 & 0.8 \\
\hline Antidepressants & 8.7 & 6.5 \\
\hline Antien & 3.4 & 1.9 \\
\hline Ant & 2.5 & 1.6 \\
\hline Other & 4.4 & 3.1 \\
\hline Others* & 0.9 & 1.0 \\
\hline Macrolide antibiotics (not on the same day of OCS) , \% & 0.20 & 0.01 \\
\hline Non-macrolide antibiotics* (not on the same day of OCS), \% & 0.4 & 0.3 \\
\hline
\end{tabular}

SD, standard deviation; NSAIDs, non steroidal anti-inflammatory drugs; COPD, chronic obstructive pulmonary disease; CNS, central nervous system; OCS, oral corticosteroids; *excluding fluoroquinolones as the exposure under study 
Table 2. Distribution of cases of serious arrhythmias by type.

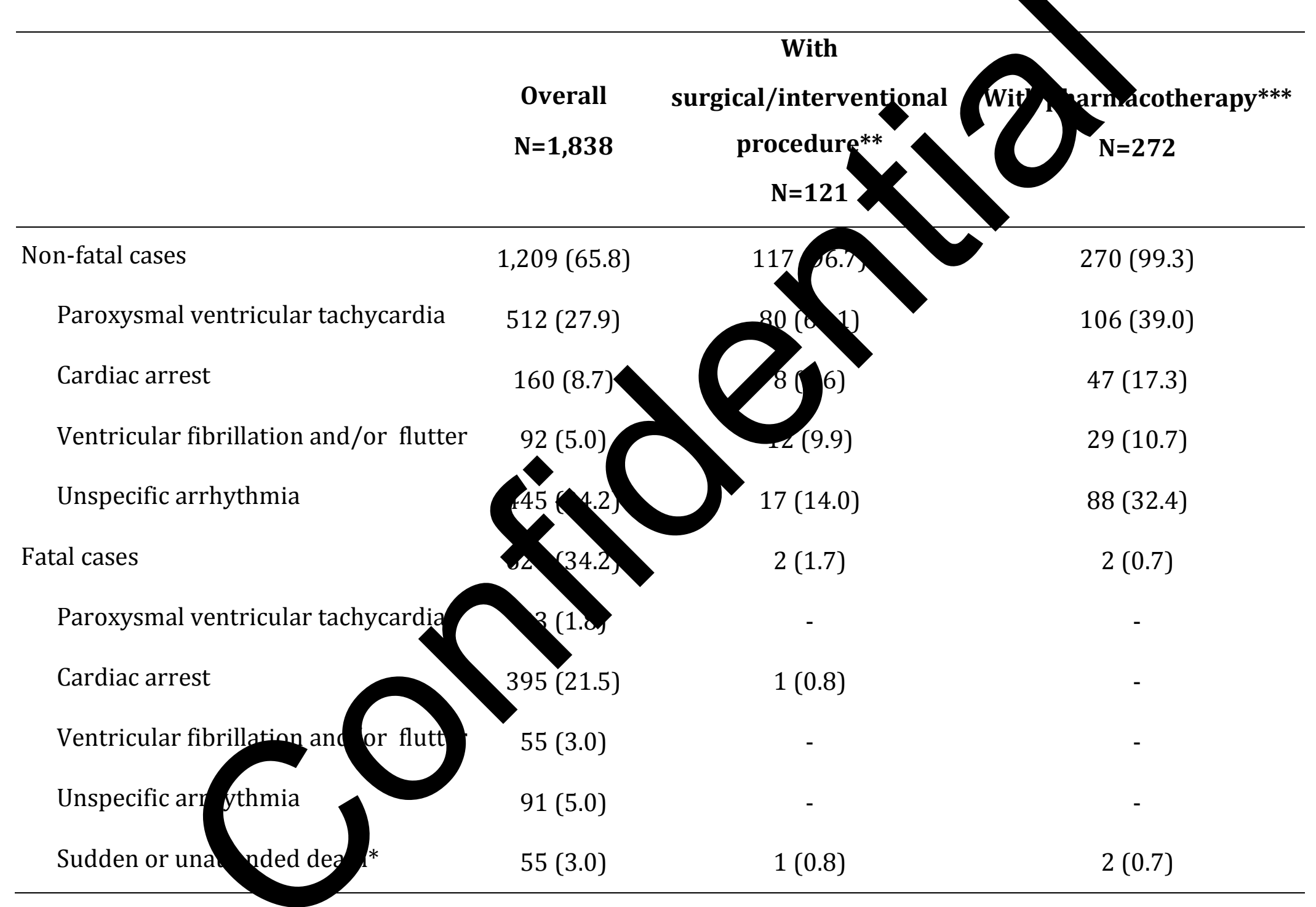

*including sudden cardiac death ${ }^{* *} \pm 3$ months within the hospitalization ${ }^{* * *} \pm 1$ months within the hospitalization 
Table 3. Crude and adjusted Rate Ratios (RRs) of serious arrhythmia for the current (within 14 days before the index date), recent (from 15 to 30 days before the index date) and past (from 31-365 days before the index do ) use of fluoroquinolones.

\begin{tabular}{|c|c|c|c|c|}
\hline & $\begin{array}{c}\text { Cases } \\
(n=1,838)\end{array}$ & $\begin{array}{c}\text { Controls } \\
(n=36,670)\end{array}$ & & Adjusted* \\
\hline \multicolumn{5}{|l|}{ Recency of use, n (\%) } \\
\hline Current, New & $20(1.1)$ & & & $1.62(0.97-2.71)$ \\
\hline Current, not New & $18(1.0)$ & & 1.75 & $1.12(0.66-1.89)$ \\
\hline All current & $38(2.1)$ & & 1.89 & $1.34(0.92-1.93)$ \\
\hline Moxifloxacin & & & 2.40 & $1.82(0.78-4.22)$ \\
\hline Levofloxacin & & & 1.66 & $0.78(0.29-2.13)$ \\
\hline Ciprofloxacin & & $245(0.7)$ & 1.78 & $1.32(0.80-2.18)$ \\
\hline Gatifloxacin & & $17(0.05)$ & 5.69 & $3.97(1.15-13.62)$ \\
\hline Others & & $46(0.1)$ & 0.93 & $0.83(0.19-3.60)$ \\
\hline Recent & $30(1.6)$ & $398(1.1)$ & 1.68 & $1.24(0.82-1.86)$ \\
\hline Past & & $4,778(13.0)$ & 1.53 & $1.14(1.00-1.32)$ \\
\hline Unexposed & $1,438(78.2)$ & $31,140(84.7)$ & Reference & Reference \\
\hline
\end{tabular}

*adjusted for all covariates reported in Table 1 
Table 4. Crude and adjusted Rate Ratios (RRs) of serious arrhythmia for the current (within 14 days before the index date), recent (from 15 to 30 days before the index date) and past (from 31-365 days before the index do ) use of fluoroquinolones, when patients hospitalized during the current time-window were excluded.

\begin{tabular}{|c|c|c|c|c|}
\hline & $\begin{array}{c}\text { Cases } \\
(n=1,649)\end{array}$ & Controls* & $\mu$ & Adjusted** \\
\hline \multicolumn{5}{|c|}{ Recency of use, n (\%) } \\
\hline Current, New & $18(1.1)$ & & 2.37 & $2.23(1.31-3.80)$ \\
\hline Current, not New & $17(1.0)$ & & 2.08 & $1.41(0.82-2.44)$ \\
\hline All current & & & 2.22 & $1.76(1.19-2.59)$ \\
\hline Moxifloxacin & & & 3.30 & $3.30(1.47-7.37)$ \\
\hline Levofloxacin & & $61(0.2)$ & 1.30 & $1.29(0.40-4.17)$ \\
\hline Ciprofloxacin & & $216(0.6)$ & 2.15 & $2.15(1.34-3.46)$ \\
\hline Gatifloxacin & & $15(0.04)$ & 7.40 & $7.38(2.30-23.70)$ \\
\hline Others & $2(0.1)$ & $44(0.1)$ & 1.05 & $1.05(0.25-4.33)$ \\
\hline Recent & $26(1.6)$ & $372(1.1)$ & 1.70 & $1.26(0.80-1.93)$ \\
\hline Past & 295 (17.9) & 4,647 (12.9) & 1.53 & $1.15(1.00-1.34)$ \\
\hline Unexposed & $1,293(78.4)$ & $30,640(85.0)$ & Reference & Reference \\
\hline
\end{tabular}


* \% are weighted for the number of controls being matched per case

**adjusted for all covariates reported in Table 1

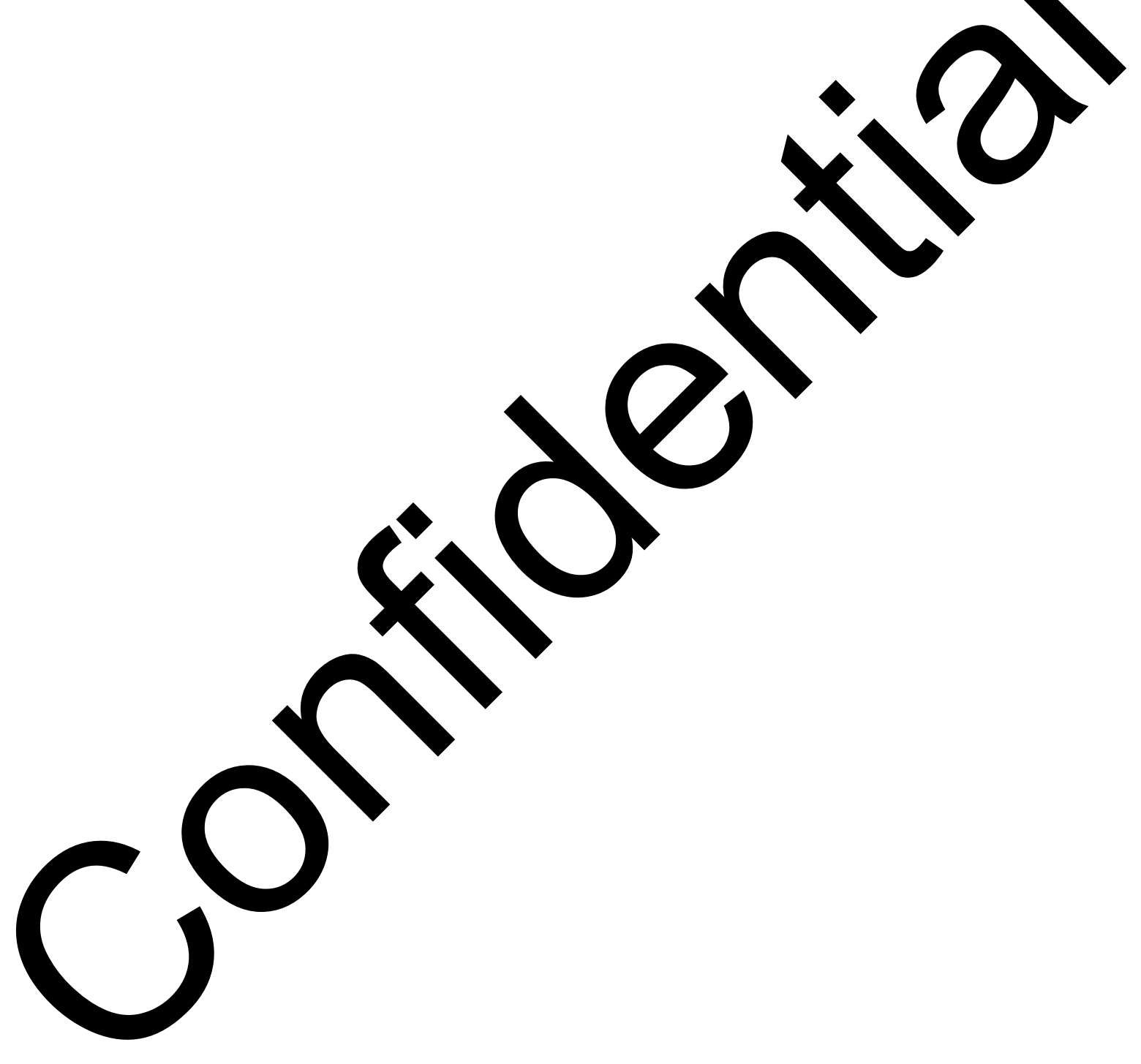


Figure (.tif and .eps files only)

Click here to download high resolution image

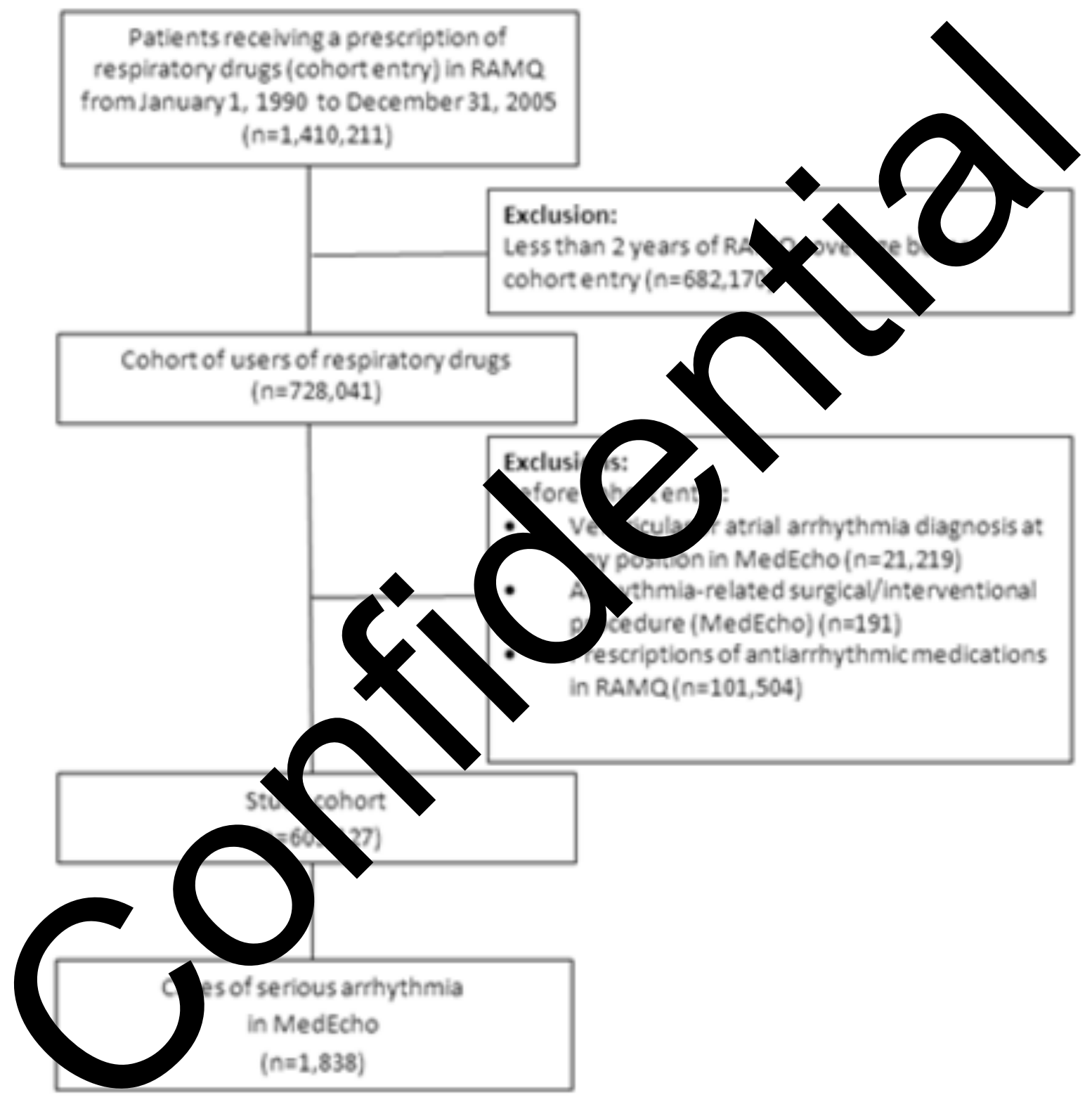

Figure 1. Study fow chart. 